\title{
Prevalence and Characteristics of Soft Tissue Calcifications in Cbct Images of Mandibular Region
}

\author{
Emine Sebnem Kursun Cakmak ${ }^{1}$ (D) Seval Bayrak ${ }^{2}$ (D) , Cemal Atakan ${ }^{3}$ \\ ${ }^{1}$ Turkiye Public Hospitals Agency, Ministry of Health, Ankara, Turkey \\ ${ }^{2}$ Abant Izzet Baysal University, Dentistry Faculty, Dentomaxillofacial Radiology Department, Bolu, Turkey \\ ${ }^{3}$ Ankara University, Faculty of Sciences, Department of Statistics, Ankara, Turkey
}

Correspondence Author: Seval Bayrak

E-mail: dtseval@hotmail.com

Received: $18.08 .2019 \quad$ Accepted: 14.11 .2019

\begin{abstract}
Objectives: The goal of the study is to evaluate the prevalence and characteristics of soft tissue calcifications (STCs) detected in the mandibular region in CBCT images.

Methods: 242 (150 female, 92 male) mandibula CBCT images were evaluated in this study. Prevalence, anatomical location, pattern (bilateral or unilateral), size $(\mathrm{mm})$, number (single or multiple), age $(<35,35-50,>50)$ and gender tendency were recorded. Images were evaluated in the axial, coronal and 3D reformated images. The Chi-square test was used to test for differences of the STC groups and the percentage values.

Results: 88 patients (36.4\%) had at least one STC in the mandibular region. The mean age of the patients with STC was $58.3 \pm 11.06$ years. No gender predilection was stated in terms of the presence of STC. STC was higher in patients over 50 years than the other age groups ( $p=0.00$ ). Most of the STCs were unilateral, above $3 \mathrm{~mm}$ in size, single and located in the lingual aspect of the mandible.

Conclusion: It is of vital importance for clinicians to know characteristics of STCs and to detect STCs in early stages especially those concerning systemic conditions and to prevent life-threatening consequences.

Keywords: Cone Beam Computed Tomography; Mandible; Soft Tissue Calcification; Prevalence
\end{abstract}

\section{INTRODUCTION}

Soft tissue calcifications (STCS) in the maxillofacial area are uncommon and generally, correspond to radiographic findings in routine radiographic examinations. STC is classified as idiopathic, dystrophic, or metastatic. Dystrophic calcification occurs in degenerating, diseased, and dead tissue despite normal serum calcium and phosphate levels. In contrast, metastatic calcification is the process by which normal undamaged tissues are calcified by means of a hypercalcemic condition like what occurs in hyperparathyroidism (1).

In the diagnosis to determine the exact location of STC is one of the primary challenges. Due to the presence of ghost images, panoramic radiographs remain incapable in the diagnosis of STC (2). In addition, STCs located in the head and neck area are positioned adjacent to each other and estimation of the true position is very difficult in conventional imaging (3).

In recent years, cone beam computed tomography (CBCT) has become more widely used in dentomaxillofacial imaging. CBCT provides three-dimensional images and supplies sagittal, coronal, and axial images plus their multiplanar transformation (4). According to the guidelines of the American Academy of Oral and Maxillofacial Radiology, CBCT images constitute a valuable tool for determining the location of STCS (5).

The goal of this retrospective study is to evaluate the prevalence of STC in the mandibular region of a Turkish subpopulation by using CBCT images and to detect their anatomical location, pattern, size, number and age/gender tendency.

\section{MATERIALS and METHODS}

The study was approved by the X University Ethical Committee (approval number 2018/15) and conducted according to the principles described in the Declaration of Helsinki, including all amendments and revisions. This retrospective study assessed the soft tissue calcification in the mandibular region of 242 patients consisting of 92 male and 150 female (from 12 to 76 years) with the mean age of 46.42 years. CBCT data 
of patients who recruited to in X University, Dentistry Faculty for several reasons were retrospectively evaluated. The CBCT images were obtained using the I-CAT 3D Imaging System (Imaging Sciences International, Hatfield, PA, USA) with the following parameters: $5 \mathrm{~mA}, 120 \mathrm{kVp}, 16 \times 13 \mathrm{~cm}$ FOV. Images were evaluated in the axial, coronal and $3 D$ reformated images. Images were evaluated according to presence, anatomical location, pattern (bilateral or unilateral), size $(\mathrm{mm})$, number (single or multiple), age $(<35,35-50,>50)$ and gender tendency. The anatomical location of STCs in relation to mandibular bone were categorized into six regions (Figure 1) as follows; Regions A and F, which comprised the vestibular aspect of the mandible, extending from the lower first premolars to the edge of the scan (distally); regions $B$ and $E$, which comprised the lingual aspect of the mandible, extending from the lower first premolars to the edge of the scan (distally); region $C$, which comprised the lingual aspect of the anterior teeth, from canine to canine; region $D$, which comprised the vestibular aspect of the anterior teeth, from canine to canine. All measurements were done twice by one observer (S.B.).The SPSS 25@ software (SPSS Inc., IBM Company Headquarters, Chicago, IL) was used for storing and analyzing data. The significance level value was set at 0.05 . The Chi-square test was used to test for differences of the STC groups and the percentage values.

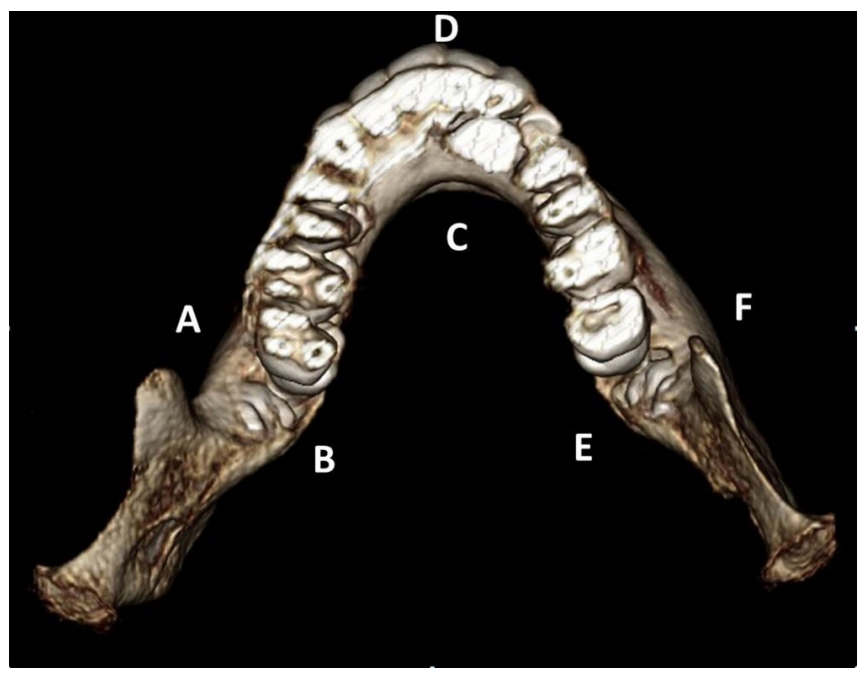

Figure 1. Schematic illustration of the location of the STC in the CBCT axial image.

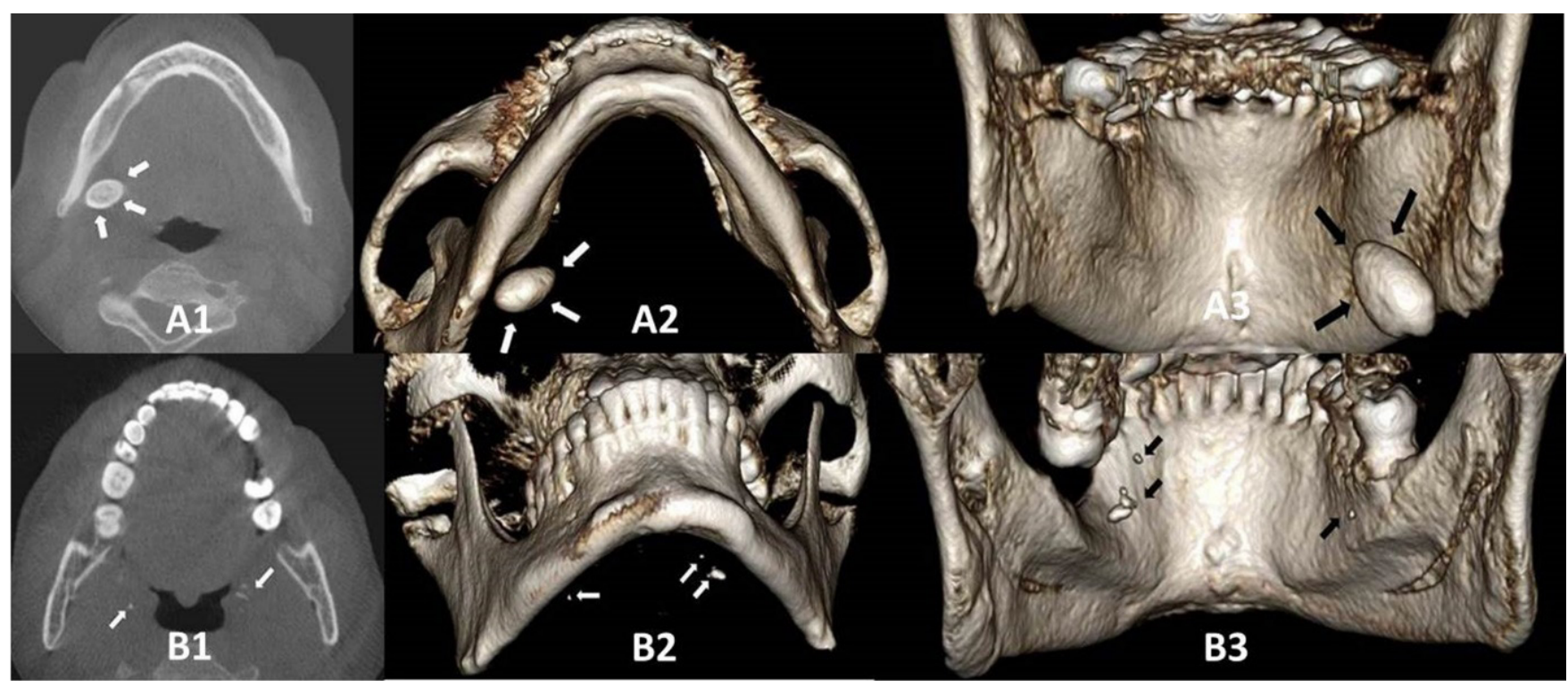

Figure 2. STC samples are shown with arrows: A1, B1 (coronal CBCT image); A2, B2 (base of mandible's 3D reconstructed image); $A 3, B 3$ (inside of the mandible's 3D reconstructed image)

\section{RESULTS}

The overall prevalence of STC was $36.4 \%$ in the studied population. The mean age of patients with STC was $58.3 \pm 11.06$ with the age ranging between 21 and 67 years. According to anatomical location, STCs were the most located in the lingual aspect of the mandible whereas the least located in the lingual aspect of the anterior teeth with statistical significance $(p=0.035)$. When we evaluated STCs in terms of pattern, unilaterality was observed higher among STCs $(p=0.000)$.
Assessment according to the size of STC showed that above 3 $\mathrm{mm}$ was the most observed size $(p=0.000)$. Multiple STCs were fewer than single STCs with statistical significance $(p=0.015)$ (Table 1). There was no statistically significant relationship between gender and the presence of the STC as demonstrated in Table $2(p=0.671)$. Age was stated statistically related to STC $(p=0.000) . S T C$ was observed higher in patients over 50 years than the other age groups (Table 3). 
Table 1. Anatomical location, pattern, size distribution and the number of the STCS.

\begin{tabular}{|c|c|c|c|c|c|c|c|c|c|c|c|}
\hline & \multicolumn{4}{|c|}{ Anatomical Location } & \multicolumn{3}{c|}{ Pattern } & \multicolumn{3}{c|}{ Size (mm) } & \multicolumn{2}{c|}{ Number } \\
\hline STC & $\mathrm{a} / \mathrm{f}$ & $\mathrm{b} / \mathrm{e}$ & $\mathrm{c}$ & $\mathrm{d}$ & Bilateral & Unilateral & $<=1$ & $1<$ size $<3$ & $>=3$ & Single & Multiple \\
\hline $\mathrm{n}$ & 26 & 35 & 3 & 24 & 27 & 61 & 28 & 25 & 35 & 55 & 33 \\
\hline$\%$ & 29.54 & 39.77 & 3.40 & 27.27 & 30.68 & 69.32 & 31.82 & 28.41 & 39.77 & 62.50 & 37.50 \\
\hline
\end{tabular}

Table 2. Correlation between gender and the presence of the STCS.

\begin{tabular}{|c|c|c|c|c|c|c|}
\hline \multicolumn{7}{|c|}{ Soft Tissue Calcifications } \\
\hline Gender & $\mathrm{n}(\%)$ & $\begin{array}{c}\text { Absent } \\
\mathrm{n}(\%)\end{array}$ & $\begin{array}{c}\text { Present } \\
\mathrm{n}(\%)\end{array}$ & Odds Ratio & $\% 95 \mathrm{Cl}$ & $p$ \\
\hline Male & $92(38.02)$ & $57(61.96)$ & $35(38.04)$ & \multirow{2}{*}{0.89} & \multirow{2}{*}{$(0.52-1.524)$} & \multirow{2}{*}{0.671} \\
\hline Female & $150(61.98)$ & $97(67.67)$ & $53(35.33)$ & & & \\
\hline Total & $242(100)$ & $154(63.63)$ & $88(36.37)$ & & & \\
\hline
\end{tabular}

Table 3: Correlation between age and the presence of the STCS.

\begin{tabular}{|c|c|c|c|c|c|c|}
\hline \multicolumn{7}{|c|}{ Soft Tissue Calcifications } \\
\hline Age & $\mathrm{n}(\%)$ & $\begin{array}{c}\text { Absent } \\
\mathrm{n}(\%)\end{array}$ & $\begin{array}{c}\text { Present } \\
\mathrm{n}(\%)\end{array}$ & Odds Ratio & $\% 95 \mathrm{Cl}$ & $p$ \\
\hline$<35$ & $57(23.55)$ & $49(31.82)$ & $8(38.04)$ & & & \multirow{3}{*}{$0.000^{*}$} \\
\hline $35-50$ & $103(42.56)$ & $62(40.26)$ & $41(35.33)$ & 4.05 & $(1.74-9.43)$ & \\
\hline$>50$ & $82(33.88)$ & $43(27.92)$ & $39(42.56)$ & 5.56 & $(2.34-13.18)$ & \\
\hline Total & $242(100)$ & $154(63.63)$ & $88(36.37)$ & & & \\
\hline
\end{tabular}

\section{DISCUSSION}

It is worth mentioning that there is a considerable variation in the prevalence of STC among various studies. The prevalence may be influenced by differences in age, studied populations and sample size. In addition, the evaluation method has great importance in the diagnosis of STCs: indeed, threedimensional imaging modalities have shown greater efficiency than conventional radiography in the detection of these conditions (6).

In the current study, the prevalence of STC was found at $36.4 \%$. Age was found to be significantly related to STC whereas gender was stated unrelated. Most of the STCS were unilateral, above $3 \mathrm{~mm}$ in size, single and located in the lingual aspect of the mandible.

The presence of STC in some tissues may be associated with the presence of a systemic condition and represent a sign of more potentially threatening consequences. It is therefore reasonable that dental practitioners to enhance their comprehension of the anatomy of the head and neck structures and potential sites of calcification (1).

da Silva Nunes et al. (2) found STC prevalence as 15\% in CBCT images of the mandible and predominantly at the posterior region of the mandible with no relation to gender and age. Icöz and Akgünlü (7) assessed 4263 panoramic radiographs for the presence of STC and estimated only $6.4 \%$ STC in the whole study population. Difference between the current study in terms of incidence may be attributed to radiological examination methods used in the studies. But in line with our study, the authors also stated that the prevalence of calcifications increased with age. Contrary to current results, the authors also detected more calcification in women than male counterparts. This relationship was stated due to a decline in the estrogen level after menopause, which affects the lipoprotein metabolism by preventing the formation of atheromatous plaques and its reduced levels may increase the prevalence (8). The limited sample size of patients to reach statistical difference between gender groups may be the limitation of the current study.

Missias et al. (6) evaluated the prevalence of STCs using CBCT images with various fields of view (FOV) including both maxilla and mandible, maxilla only and mandible only. Of the studied samples, STC was observed in $62.6 \%$ with the highest prevalence in both maxilla and mandible (76.8\%) and the least prevalence in maxilla only (57.2\%). The authors found no association between STCs and patients' gender or age.

Khojastepour et al. (3) found the prevalence of STC as 25.9 $\%$ in the mandibular region with the mean age 51.7 years. STCs were higher in the male population and predominantly detected in the posterior region of the mandible. The prevalence of STC increased with age and most of them were smaller than $3 \mathrm{~mm}$. In the current study, in contrast with Khojastepour et al. the rate of incidence was higher (36.4\%), the mean age of patients with STC was higher, no gender predilection was stated and most of the STCS were higher than $3 \mathrm{~mm}$.

In a study by Garay et al. (9) 3028 dental panoramic radiographs were examined in terms of STC in the mandibular 
angle area. They stated a total of 79 calcifications (2.61\%) in 75 individuals which increases with age. Also, a large number of the calcifications found were small, of low density and unilateral.

The other limitation of this study is the retrospective nature that makes it impossible to clinically examine, to reach patients' entire history and to accurately diagnose the patients. So we prefer not to classify calcifications themselves. We also have not compared our results with the studies those reporting the incidence of various STCs such as tonsilloliths, sialoliths.

\section{CONCLUSION}

In the current study, a considerable amount of STC was detected in the mandibular region especially in the older age group. Considering these finding it is essential for the practitioner to have detailed knowledge regarding these entities.

\section{REFERENCES}

[1] Çağlayan F, Sümbüllü MA, Miloğlu Ö, Akgül HM. Are all soft tissue calcifications detected by cone-beam computed tomography in the submandibular region sialoliths? J Oral and Maxillofac Surg 2014;72:1531. e1-. e6.
[2] Nunes LFdS, Santos KCP, Junqueira JLC, Oliveira JX. Prevalence of soft tissue calcifications in cone beam computed tomography images of the mandible. Revista Odonto Ciência 2011;26:297-303.

[3] Khojastepour L, Haghnegahdar A, Sayar H. Prevalence of soft tissue calcifications in CBCT images of mandibular region. J Dent 2017;18:88.

[4] Krzyżostaniak J, Kulczyk T, Czarnecka B, Surdacka A. A comparative study of the diagnostic accuracy of cone beam computed tomography and intraoral radiographic modalities for the detection of noncavitated caries. Clin Oral Investig 2015;19:667-72.

[5] Scarfe W, Farman A. Soft tissue calcifications in the neck: Maxillofacial CBCT presentation and significance. AADMRT Currents 2010;2:3-15.

[6] Missias E, Nascimento E, Pontual M, Pontual A, Freitas D, Perez D, et al. Prevalence of soft tissue calcifications in the maxillofacial region detected by cone beam CT. Oral Diseases 2018;24:628-37.

[7] Icoz D, Akgunlu F. Prevalence of detected soft tissue calcifications on digital panoramic radiographs. SRM J Res Dent Sci 2019;10:21.

[8] Juliana-Mara-Oliveira Santos G-C, Soares A-P-N-NA, LúcioMitsuo Kurita P-G-d, Barros Silva F-W-GC. Prevalence of carotid artery calcifications among 2,500 digital panoramic radiographs of an adult Brazilian population. Med Oral, Pat Oral y Cirugia Bucal 2018;23:e256.

[9] Garay I, Netto HD, Olate S. Soft tissue calcified in mandibular angle area observed by means of panoramic radiography. Int J of Clin and Exp Med 2014;7:51. 